\title{
Single Dose Acute Toxicology in a Preclinical Trial: The Basic Step in Drug Discovery and Development
}

\author{
Yilkal Belay* \\ School of Biomedical Sciences, College of Health Sciences, Makerere University, Kampala, Uganda \\ ^Corresponding author: Yilkal Belay, School of Biomedical Sciences, College of Health Sciences, Makerere University, Kampala, Uganda; Email: tarikuyilkal@yahoo.com
}

Received: November 24, 2021; Accepted: November 30, 2021; Published: December 05, 2021

\section{Extended Abstract: 21 $1^{\text {st }}$ European Biotechnology congress 2018, Moscow, Russia}

The dose in a preclinical trial literally refers to the amount of a test compound that has to be administered to a study subject to evaluate its pharmacological suitability. Depending on the objective of the trial, different levels of doses have to be prepared and separately administered to the study animals to be able to determine the suitable dose of a test compound for the next phase of a trial. There is, however, scientific misconception about the role of a dose in experimental pharmacology in which it is considered to be the fundamental concept of toxicology that avoids the poison of a test compound which is far from scientific reality due to the fact that the nature of a test compound could not be changed by simply quantification. The natural property of a compound could neither be changed nor eliminated by limiting the amount of a dose that has to be administered to a study animal. It is a contradiction to the scientific law of physics which states that "matter can neither be created nor destroyed but rather it can be transformed into other form of matter by the use of energy". The basic principle of toxicology is, however, deviated from this scientific reality by the fact that it judges to create a compound with different pharmacological property from a single test compound by simply quantification. In other words, it uses a hypothetical concept in experimental pharmacology in which the lower dose is considered to be safe when the higher dose of the same test compound is unsafe for life. It is important to note that only one molecule of a test compound binds with the binding domain of a drug receptor to trigger a biological signal. The pharmacologic property of one molecule of a test compound, therefore, could not be different from the pharmacologic property of multiple molecules of the same compound despite the magnitude of a biological response that could be able to manifest on study animals within the shortest possible time in the course of metabolism. This means that one molecule of a test compound could trigger the same physio-pharmacological mechanisms as ten molecules of the same test compound within the biological processes of an organism. However, the magnitude of a biological response against ten molecules of a test compound could be ten times higher than the magnitude of a biological response of one molecule of the same test compound administered to study animals with similar biological functionality and strength of natural immunity. If we administered a higher dose to a study animal, we could easily notice a response in a short period of time as compared to a lower dose of the same test compound. The adverse effect of a dose of a test compound administered to a study animal is directly proportional to the magnitude of immunoglobulins immune response against its harmful molecules [1]. Immunoglobulins are cell signalling proteins embedded in the cell membrane with the ability to detect the harmful molecules of a test compound against which it responds by activating B lymphocytes to proliferate and produce new immunoglobulin molecules. Immunoglobulins also exist freely in the plasma but it does not involve in cell signalling and cell activation mechanisms [2]. The amount of newly formed immunoglobulins would increase in blood serum as the number of harmful molecules of a test compound interacted with signalling proteins has also increased except with a test compound that has a depressant effect on the metabolic system of an organism that could also depress the amount of immunoglobulin molecules in blood serum as the immune and metabolic systems are directly interrelated. However, the amount of immunoglobulins in blood serum usually declines when the toxic severity of a dose administered to a study animal has reached at its peak in which the signalling mechanisms of immunoglobulins seemingly desensitised as the number of toxic molecules of a dose interacted with signalling proteins has increased [1]. Thus, immunoglobulins response is crucial in experimental pharmacology and toxicology to determine the toxic severity of a dose that is again enabling us to decide the safety pharmacology of a test compound. The dose of a test compound is said to be safe when the magnitude of its toxic severity is $\leq 0$.

The previous studies conducted in 2011 and 2019 have shown that the pharmacological property of any amount of administered test material into study Balb $\mathrm{c}$ mice remained intact whether it was high or very low in amount [1-3]. The amount of administered dose, however, changed the magnitude of a biological response and the length of time at which undesired effect was manifested on Balb c mice treated orally. The pharmacological effect of a dose starts at the biochemical and molecular level of exposed organism which perhaps cause biological response at the cellular level which eventually leads to biological response at the organismal level as the reactive dose in the natural process of an organism increases all of which has regulatory mechanism at each level [4]. The biological effect of lower doses perhaps limited at the molecular level which impacts the health of exposed organisms in the long run as genetic disorders or metabolic disorders or cancer of different types depending on the site of damage 
introduced to the biological system. Disease such as cancer may result from an abnormality in function or structure of a single cell induced by a dose of noxious chemicals. This implies to the fact that the amount of a dose could not avoid or eliminate the harmful property of a drug that has to be administered to a study animal. In the previous experimental studies, all tested chemicals were toxic at any amount with different intensity which was computed using biological responses as toxic reaction rate and toxic severity during the course of metabolism [1,3]. This biological approach was considered one independent and two dependant research variables in order to be able to compute toxic severity and toxic reaction rate of a dose administered to study Balb c mice orally. The independent and dependent research variables respectively used were: [3], the administered dose, [1], elapsed time for the manifestation of recognisable adverse effect in the biological system of treated Balb c mice and [3], the changes in the amount of immunoglobulins in blood serum after dosing. The pharmacological property of tested chemicals were determined by the computed result of both toxic reaction rate and toxic severity rather than by the amount of a test chemical that has been administered to the study Balb $c$ mice. The toxic reaction rate refers to the number of harmful molecules of administered dose that has been interacted with its receptor and has manifested undesired biological response on a study animal which was computed using a mathematical formula $\left(r=\frac{d}{t}-\Delta I g\right) \mathrm{mg} / \mathrm{sec}$ whereas the toxic severity refers to the magnitude of a biological harm or injury caused by the dose of a drug that has been administered to a study animal which was also computed using a mathematical formula $\left(s=\frac{r}{d} \times 100\right) \% /$ sec where $r$ is toxic reaction rate, $s$ is toxic severity, $d$ is administered dose, $t$ is elapsed time for adverse effect manifestation and $\Delta I g$ is the changes in immune response after dosing. The study has revealed that the toxic severity of a dose was the reason for the limited lifespan of treated animals whereas the toxic reaction rate accounted for the safety pharmacology of tested chemicals. The higher the amount of a dose administered to a study animal, the higher the toxic severity was that had influenced the lifespan of exposed Balb c mice. This implies to the fact that the dose doesn't determine safety but rather lifespan of study animals in its natural environment. This means that the harmful effect of a test chemical within the biologic process of an organism is determined by the chemical nature rather than by the amount of a dose administered. The amount of a dose, however, could speed up the time at which biochemical and physio-pathological changes would be manifested on treated animals. Since the higher dose could manifest undesirable biological response within a short period of time and the lower dose after a long period of time on treated animals, categorising of a single test chemical into minimum lethal dose $\left(\mathrm{LD}_{50}\right)$ and maximum effective does $\left(\mathrm{ED}_{50}\right)$ has no scientific ground to declare at a point of time that the lower dose is safe and the higher dose is unsafe for life. The undesirable biological effect of lower doses of a test compound is likely to be manifested in the late ages of an organism which could be the reason why cancer is more prevalent in the elderly population. The etiologic agent perhaps introduced to our biological process in early ages and its undesired effect possibly manifested in the late ages. This means that if the higher dose is lethal to a study animal, there is no scientific reason to declare that the lower dose is safe. A test compound is said to be toxic not only when it has caused death but also undesirable biological mechanism in the study animals.
Evolution has showed that all living things inherited desirable and typical genetic material from their predecessors through reproduction which naturally makes the difference among themselves [5]. Today, however, there are thousands and millions of humans and animals with anomalies and disabilities which might be either hereditary or nonhereditary depending on the site of damage introduced into the cell, tissue or organ system in which drug is one of the highest risk factors for the incidence. Therapeutic drugs such as valproic acid, thalidomide and warfarin have been proved to be teratogens after being on market for many years [4]. There are thousands and millions of other diseases caused by chromosomal abnormalities and gene defects such as Cri du chat syndrome, Down syndrome and Achondroplasia, fragile-x syndrome respectively [5]. The genetic changes that causes these diseases can be a whole additional chromosome or a whole missing chromosome or a change of a single base in a gene sequence [5]. However, there is no specifically defined cause, other than speculation, about the abnormal chromosomes and defected genes which are causing these diseases. There are many chemical agents that can cause damage to the nucleus of a cell and other cell organelles such as adverse effects of prescribed medications, poisons, environmental pollutants and recreational drugs like alcohol which are high risk factors for genetic disorders causing these diseases. In general, the drug's mode of damaging the biological structure of an organism is diverse depending on the diverse chemical nature of the drug and nature of biological component of an organism responded to it. The undesirable effect of a drug might be manifested at the biochemical, cellular or organismal level depending on the amount of administered drug in which categorizing a single test material into minimum lethal dose $\left(\mathrm{LD}_{50}\right)$ and maximum effective dose $\left(\mathrm{ED}_{50}\right)$ could not ensure the safety pharmacology of any test drug.

\section{References}

1. Yilkal Tariku Belay (2019) Study of the principles in the first phase of experimental pharmacology: The basic step with assumption hypothesis. BMC Pharmacology and Toxicology.

2. Schroeder H. and Cavacini (2013) Structure and function of the immunoglobulins, $J$ Allergy Clin Immunol 125: S41-S52. [crossref]

3. Belay Y (2011) Study of safety and effectiveness of traditional dosage forms of the seed of Aristolochia elegans mast against malaria and laboratory investigation of pharmaco-toxicological properties and chemical constituents of its crude extracts. Ann Trop Med Public Health 4: 33-41.

4. Yilkal Tariku Belay (2019) Misconception about the role of a dose in pharmacology, Short review report on the biological and clinical effects. Adv Bioeng Biomed Sci Res $(A B B R) 2$

5. Huelsenbeck JP, Ronquist F, Nielsen R, Bollback (2001) Bayesian Inference of Phylogeny and Its Impact on Evolutionary Biology. Science 294: 2310-2314. [crossref]

\section{Citation:}

Belay Y (2021) Single Dose Acute Toxicology in a Preclinical Trial: The Basic Step in Drug Discovery and Development. J Pharmacol Pharm Res Volume 4(4): 1-2. 